\title{
Paper
}

\section{Some remarks on the instability of approximate solutions for ODEs}

\author{
Takuma Kimura $^{1 a)}$, Takehiko Kinoshita ${ }^{2}$, \\ and Mitsuhiro T. Nakao ${ }^{3}$ \\ ${ }^{1}$ JST CREST/Faculty of Science and Engineering, Waseda University, \\ Tokyo 169-8555, Japan. \\ ${ }^{2}$ RIMS, Kyoto University, \\ Kyoto 606-8502, Japan. \\ ${ }^{3}$ Sasebo National College of Technology, \\ Nagasaki 857-1193, Japan. \\ a) tkimura@aoni.waseda.jp
}

Received April 10, 2012; Revised July 24, 2012; Published January 1, 2013

\begin{abstract}
We show a strange property of an approximate solution for the initial value problem of ODEs. This study is originally motivated by the numerical verification methods of solutions for parabolic initial-boundary value problems. We prove that an a priori norm estimate of the derivative of approximation for simple initial value problems could not be stable, even though the concerned numerical scheme is a natural finite element approximation. Since the corresponding estimates for the exact solution is bounded by a given function, it implies that the discretization could no longer maintain the same property at all. Therefore, this result should be an interesting example which shows an essential gap between the infinite and finite dimensional problems.
\end{abstract}

Key Words: a priori estimates, Galerkin methods, linear ordinary differential equation

\section{Introduction}

In $[2,3]$, the authors proposed computational methods of a constant $C_{\mathcal{L}_{t}^{-1}}$ for the following linear parabolic initial-boundary value problems:

$$
\begin{cases}\frac{\partial}{\partial t} w-\nu \Delta w+(b \cdot \nabla) w+c w=g, & \text { in } \Omega \times J, \\ w(x, t)=0, & \text { on } \partial \Omega \times J, \\ w(x, 0)=0, & \text { in } \Omega,\end{cases}
$$

satisfying

$$
\|w\|_{L^{2}\left(J ; H_{0}^{1}(\Omega)\right)} \leq C_{\mathcal{L}_{t}^{-1}}\|g\|_{L^{2}\left(J ; L^{2}(\Omega)\right)} .
$$

Here, $t \in J=(0, T] \subset \mathbb{R}, x \in \Omega \subset \mathbb{R}^{d}, \nu \in \mathbb{R}$ a positive consatant, $b \in L^{\infty}\left(J ; L^{\infty}(\Omega)\right)^{d}, c \in$ $L^{\infty}\left(J ; L^{\infty}(\Omega)\right), g \in L^{2}\left(J ; L^{2}(\Omega)\right)$. The estimates (1) are derived by using a full discrete scheme in 
[2] and semi-discretization only for space direction in [3] for the simple heat equation of the form: $\frac{\partial}{\partial t} u-\nu \Delta u=g$. Particularly, [2] used the finite element subspace defined by $S_{h}^{k} \equiv S_{h}(\Omega) \otimes S^{k}(J)$, where $S_{h}(\Omega)$ stands for a finite element subspace of $H_{0}^{1}(\Omega)$ and $S^{k}(J)$ for $H^{1}(J)$, respectively. And they defined the following full discretization $u_{h}^{k} \in S_{h}^{k}$ by

$$
\left(\frac{\partial}{\partial t} u_{h}^{k}, v_{h}^{k}\right)_{L^{2}\left(J ; L^{2}(\Omega)\right)}+\nu\left(u_{h}^{k}, v_{h}^{k}\right)_{L^{2}\left(J ; H_{0}^{1}(\Omega)\right)}=\left(g, v_{h}^{k}\right)_{L^{2}\left(J ; L^{2}(\Omega)\right)},{ }^{\forall} v_{h}^{k} \in S_{h}^{k}
$$

and used the estimates

$$
\left\|\frac{\partial}{\partial t} u_{h}^{k}\right\|_{L^{2}\left(J ; L^{2}(\Omega)\right)} \leq \sigma\|g\|_{L^{2}\left(J ; L^{2}(\Omega)\right)} .
$$

In the above estimates, however, we observed some unexpected phenomena such that $\sigma \rightarrow \infty$ as $k \rightarrow 0$, which should be a disadvantage for the computation of $C_{\mathcal{L}_{t}^{-1}}$. Taking notice of the semidiscretization of the heat equation, it is seen that the full discrete approximation $u_{h}^{k}$ is represented by an approximate solution of the following system of ODEs

$$
\left\{\begin{array}{l}
\frac{d \mathrm{u}}{d t}+B \mathrm{u}=\mathrm{g}, \quad \text { in } J \\
\mathrm{u}(0)=0
\end{array}\right.
$$

where $B \in L^{\infty}(J)^{n \times n}, \mathrm{~g} \in L^{2}(J)^{n}$. We also observed similar phenomena even for the following simple initial value problem of single ODE, which is a direct motivation of the present study.

$$
\left\{\begin{array}{l}
\frac{d}{d t} u(t)+\kappa u(t)=f(t), \quad t \in J, \\
u(0)=0,
\end{array}\right.
$$

where $J=(0, T]$ is a bounded interval with fixed $T>0, f \in L^{2}(J)$ a given function, $\kappa \in \mathbb{R}^{1}$ a parameter.

Let $u$ be an exact solution and $u_{h}$ an approximate solution of (2), respectively. Here, $h$ means a discretization step size used to compute $u_{h}$.

Then we consider a priori estimates of the form

$$
\begin{gathered}
\left\|u^{\prime}\right\|_{L^{2}(J)} \leq C^{*}\|f\|_{L^{2}(J)}, \\
\left\|u_{h}^{\prime}\right\|_{L^{2}(J)} \leq C\|f\|_{L^{2}(J)},
\end{gathered}
$$

where $C^{*}$ and $C$ are positive constants independent of $f$ and $h$, but might depend on $\kappa$ and $T$.

In this paper, we show an example which proves non-existence of such a constant $C$ in (4) for $u_{h}$ computed by a natural finite element approximation, while there exists $C^{*}$ in (3). It implies that the original property no longer holds for the finite element approximation, namely, there exists an essential gap between the infinite and finite dimensional problems.

\subsection{Notation}

We denote by $L^{2}(J)$ and $H^{1}(J)$ the usual Lebesgue and Sobolev spaces on $J$, respectively, and set

$$
(u, v)_{L^{2}(J)}:=\int_{0}^{T} u(t) v(t) d t,
$$

the inner product of $u, v$ in $L^{2}(J)$. By considering the initial condition, we put following subspace of $H^{1}(J)$ as

$$
V^{1}(J):=\left\{u \in H^{1}(J) ; u(0)=0\right\} .
$$

$V^{1}(J)$ is a Hilbert space with the inner product

$$
(u, v)_{V^{1}(J)}:=\left(\frac{d u}{d t}, \frac{d v}{d t}\right)_{L^{2}(J)} .
$$

Now, let $S_{h} \equiv S_{h}(J) \subset V^{1}(J)$ be a finite element subspace dependent on the mesh size $h$. Let $n$ be the degree of freedom for $S_{h}(J)$ and let $\left\{\phi_{i}\right\}_{i=1}^{n} \subset V^{1}(J)$ be a basis of $S_{h}(J)$. We define the $L^{2}$-projection $P_{h}^{0}: L^{2}(J) \rightarrow S_{h}(J)$ by the following variational equation

$$
\left(u-P_{h}^{0} u, v_{h}\right)_{L^{2}(J)}=0, \quad \forall v_{h} \in S_{h}(J) .
$$




\section{Estimates for exact solutions}

In this section, we consider some theoretical results on the estimates (3).

Lemma 2.1. For any $f \in L^{2}(J)$, the following a priori estimate holds

$$
\left\|u^{\prime}\right\|_{L^{2}(J)} \leq\left(\frac{1}{2}\left(e^{-2 \kappa T}+2 \kappa T-1\right)^{1 / 2}+1\right)\|f\|_{L^{2}(J)} .
$$

Proof. The exact solution of (2) can be written as,

$$
u(t)=\int_{0}^{t} e^{\kappa(s-t)} f(s) d s .
$$

Therefore, we have

$$
\begin{aligned}
\left\|u^{\prime}\right\|_{L^{2}(J)} & =\|-\kappa u+f\|_{L^{2}(J)} \\
& \leq|\kappa|\|u\|_{L^{2}(J)}+\|f\|_{L^{2}(J)} \\
& =|\kappa|\left\|\int_{0}^{t} e^{\kappa(s-t)} f(s) d s\right\|_{L^{2}(J)}+\|f\|_{L^{2}(J)} \\
& \leq\left(\frac{1}{2}\left(e^{-2 \kappa T}+2 \kappa T-1\right)^{1 / 2}+1\right)\|f\|_{L^{2}(J)} .
\end{aligned}
$$

In case of $\kappa \geq 0$, we have following estimates.

Lemma 2.2. If $\kappa \geq 0$, then for any $f \in L^{2}(J)$, the following a priori estimate holds

$$
\left\|u^{\prime}\right\|_{L^{2}(J)} \leq\|f\|_{L^{2}(J)} .
$$

Proof. By (2), we have

$$
\left(u^{\prime}+\kappa u, v^{\prime}\right)_{L^{2}(J)}=\left(f, v^{\prime}\right)_{L^{2}(J)}, \quad{ }^{\forall} v \in V^{1}(J) .
$$

From $\kappa \geq 0$ and (6), observe that

$$
\begin{aligned}
\left\|u^{\prime}\right\|_{L^{2}(J)}^{2} & \leq\left\|u^{\prime}\right\|_{L^{2}(J)}^{2}+\frac{\kappa}{2} u(T)^{2} \\
& =\left(u^{\prime}, u^{\prime}\right)_{L^{2}(J)}+\kappa\left(u, u^{\prime}\right)_{L^{2}(J)} \\
& =\left(u^{\prime}+\kappa u, u^{\prime}\right)_{L^{2}(J)} \\
& =\left(f, u^{\prime}\right)_{L^{2}(J)} \\
& \leq\|f\|_{L^{2}(J)}\left\|u^{\prime}\right\|_{L^{2}(J)}
\end{aligned}
$$

which proves the lemma.

Lemma 2.1 shows the existence of a constant $C^{*}$. Particularly, if $\kappa \geq 0$, then Lemma 2.2 gives a more accurate $C^{*}$ than Lemma 2.1 .

\section{Estimates for approximate solutions}

Note that the problem (2) is equivalently defined as the following variational form

$$
\left(u^{\prime}+\kappa u, v\right)_{L^{2}(J)}=(f, v)_{L^{2}(J)}, \quad{ }^{\forall} v \in V^{1}(J) .
$$

Therefore, it will be quite natural to define the approximate solution $u_{h} \in S_{h}$ by

$$
\left(u_{h}^{\prime}+\kappa u_{h}, v_{h}\right)_{L^{2}(J)}=\left(f, v_{h}\right)_{L^{2}(J)}, \quad{ }^{\forall} v_{h} \in S_{h} .
$$


Now, we consider the estimate of $u_{h}^{\prime}$. Let $f_{h} \in S_{h}$ be the $L^{2}$-projection of $f$.

Since $u_{h}, f_{h} \in S_{h}$, these can be written as the linear combination of the basis functions, i.e., there exist $a, b \in \mathbb{R}^{n}$ such that,

$$
u_{h}=\sum_{i=1}^{n} a_{i} \phi_{i}, \quad f_{h}=\sum_{i=1}^{n} b_{i} \phi_{i}
$$

We define $L, R, D, G \in \mathbb{R}^{n \times n}$ and $g \in \mathbb{R}^{n}$ by

$$
\begin{gathered}
L_{i j}:=\left(\phi_{i}, \phi_{j}\right)_{L^{2}(J)}, \quad R_{i j}:=\left(\phi_{i}, \phi_{j}^{\prime}\right)_{L^{2}(J)}, \quad D_{i j}:=\left(\phi_{i}^{\prime}, \phi_{j}^{\prime}\right)_{L^{2}(J)}, \\
G:=R+\kappa L, \quad g_{i}:=\left(\phi_{i}, f\right)_{L^{2}(J)}
\end{gathered}
$$

for each $1 \leq i, j \leq n$. We assume that $G$ is a nonsingular matrix. Note that $L$ and $D$ are symmetric positive definite matrices, and that $R$ is a nonsingular matrix. We denote a Cholesky factor of $L$ by $L^{1 / 2}$ such that

$$
L=L^{1 / 2} L^{T / 2}, \quad L^{T / 2}=\left(L^{1 / 2}\right)^{T} .
$$

By a similar way as in [7], we have the following estimate.

Lemma 3.1. Setting $C(h):=\left\|L^{T / 2} G^{-T} D G^{-1} L^{1 / 2}\right\|_{2}^{1 / 2}$, it holds that, for any $f \in L^{2}(J)$,

$$
\left\|u_{h}^{\prime}\right\|_{L^{2}(J)} \leq C(h)\|f\|_{L^{2}(J)} .
$$

Proof. From (9), we have,

$$
\left\|u_{h}^{\prime}\right\|_{L^{2}(J)}^{2}=a^{T} D a, \quad\left\|f_{h}\right\|_{L^{2}(J)}^{2}=b^{T} L b .
$$

Moreover, from (8), (9) and the definition of $f_{h}$, we obtain

$$
G a=g, \quad L b=g
$$

and

$$
\left\|f_{h}\right\|_{L^{2}(J)} \leq\|f\|_{L^{2}(J)}
$$

Consequently, we obtain,

$$
\frac{\left\|u_{h}^{\prime}\right\|_{L^{2}(J)}^{2}}{\|f\|_{L^{2}(J)}^{2}} \leq \frac{\left\|u_{h}^{\prime}\right\|_{L^{2}(J)}^{2}}{\left\|f_{h}\right\|_{L^{2}(J)}^{2}}=\frac{g^{T} G^{-T} D G^{-1} g}{g^{T} L^{-1} g} .
$$

Therefore, we have the estimate

$$
\begin{aligned}
\frac{\left\|u_{h}^{\prime}\right\|_{L^{2}(J)}^{2}}{\|f\|_{L^{2}(J)}^{2}} & \leq \sup _{g \neq 0} \frac{g^{T} G^{-T} D G^{-1} g}{g^{T} L^{-1} g}, \\
& =\left\|L^{T / 2} G^{-T} D G^{-1} L^{1 / 2}\right\|_{2},
\end{aligned}
$$

which implies the lemma.

Remark 3.2. From the above proof, it is readily seen that $\left\|L^{T / 2} G^{-T} D G^{-1} L^{1 / 2}\right\|_{2}^{1 / 2}$ is the best constant for which the inequality (10) holds for any $f \in L^{2}(J)$ (cf. [6]). 


\subsection{Numerical experiments}

Since the actual behavior of the estimates for $C$ in Lemma 3.1 is still not clear, we try to estimate it numerically. We use $S_{h}$ defined by piecewise linear basis functions on $J$ with $T=1$. Figure 1 shows the upper bounds of $\left\|L^{T / 2} G^{-T} D G^{-1} L^{1 / 2}\right\|_{2}$ for various mesh size $h$ and parameter $\kappa$ drawn by log-log scales. Computations are carried out on a Dell Precision T7500 (Intel Xeon x5680, 72GB of memory) with MATLAB R2010a. The computational errors have been taken into account by using INTLAB 6.0, a toolbox for self-validating algorithms, developed by Rump [5]. The values of matrix 2 norm are computed by the validated method proposed in [4].

If $\kappa=0$, then the behavior of $u_{h}^{\prime}$ is expected to be very close to $f_{h}$ for small $h$. However, as in Fig. 1, actual estimates of $\sup _{0 \neq f \in L^{2}} \frac{\left\|u_{h}^{\prime}\right\|_{L^{2}(J)}^{2}}{\|f\|_{L^{2}(J)}^{2}}$ looks like $O\left(h^{-1}\right)$ whether $\kappa=0$ or not. These numerical evidence strongly suggests that the constant $C$ in (4) could not exist.

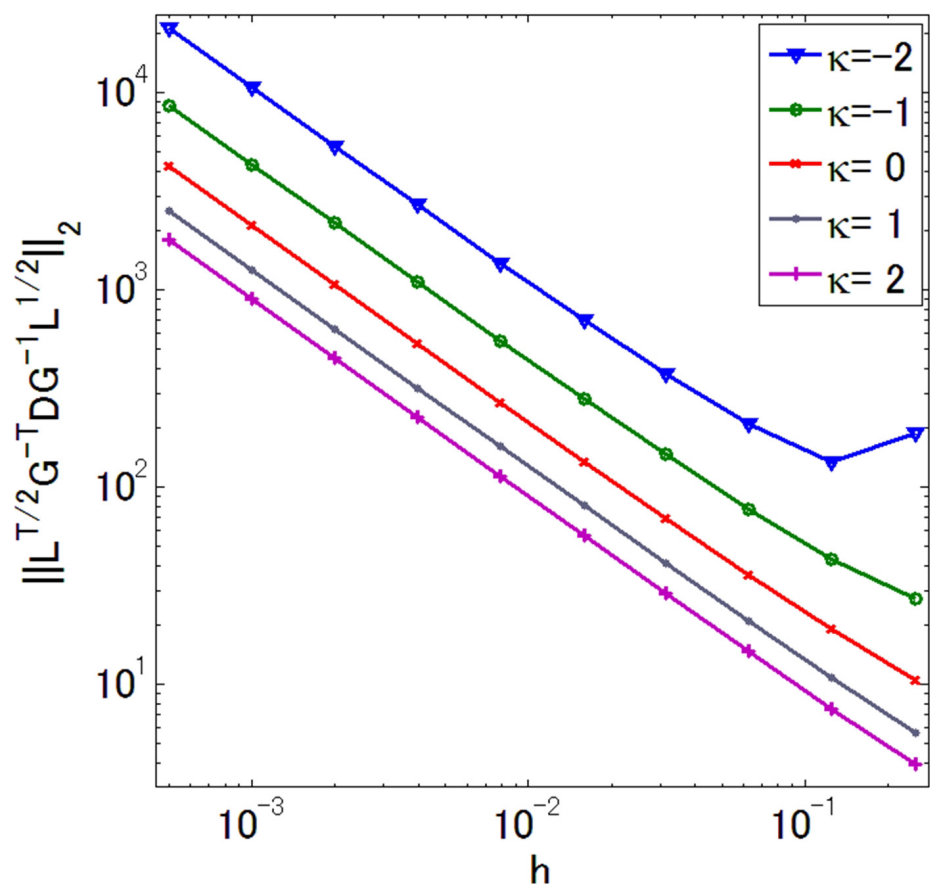

Fig. 1. Estimates by Lemma $3.1(T=1)$.

\section{Non-existence of $C$}

In this section, we theoretically exemplify that,

$$
\sup _{0 \neq f \in L^{2}(J)} \frac{\left\|u_{h}^{\prime}\right\|_{L^{2}(J)}}{\|f\|_{L^{2}(J)}} \rightarrow \infty \quad \text { as } \quad h \rightarrow 0
$$

which proves the non-existence of the constant $C$, independent of $h$, in (4).

Now let $0=t_{0}<\cdots<t_{n}=T$ be the mesh points on $J$ uniformly distributed with mesh size $h=t_{i}-t_{i-1}(i=1, \cdots, n)$, and let $S_{h}$ be the associated piecewise linear finite element space on $J$. Here, we assume that $n$ is an odd number( we can also similarly prove for an even number). We define the $u \in S_{h}$ and then set $f \in L^{2}(J)$ by

$$
u:=\phi_{1}+\phi_{3}+\phi_{5}+\cdots+\phi_{n-2}+\phi_{n} \quad \text { and } \quad f \equiv u^{\prime}+\kappa u,
$$

where $\left\{\phi_{i}\right\}_{1 \leq i \leq n}$ is the basis functions satisfying,

$$
\phi_{i}\left(t_{j}\right)= \begin{cases}1, & i=j \\ 0, & i \neq j\end{cases}
$$


Since $u \in S_{h}$, we have

$$
u=P_{h}^{0} u=u_{h},
$$

where $u_{h}$ means the solution of (8) with right-hand side $f$ defined above. Moreover, the definition of $L^{2}$-projection leads

$$
\begin{aligned}
f_{h} & \equiv P_{h}^{0} f \\
& =P_{h}^{0}\left(u^{\prime}+\kappa u\right) \\
& =P_{h}^{0}\left(u^{\prime}\right)+\kappa u_{h} .
\end{aligned}
$$

\subsection{For the case : $\kappa=0$}

First, we consider in case of $\kappa=0$ for simplicity. In this case,

$$
f=u_{h}^{\prime} \quad \text { and } \quad f_{h}=P_{h}^{0}\left(u^{\prime}\right)
$$

hold. Figure 2 and Figure 3 show the graphs of the functions $u$ and $f$, respectively.

Theorem 4.1. If $\kappa=0$, then the functions $u$ and $f$ defined by (15) have the following property

$$
\frac{\left\|u_{h}^{\prime}\right\|_{L^{2}(J)}}{\left\|f_{h}\right\|_{L^{2}(J)}}=\left(\frac{2 T}{\sqrt{3} h} \frac{(2+\sqrt{3})^{n}+(2-\sqrt{3})^{n}}{(2+\sqrt{3})^{n}-(2-\sqrt{3})^{n}}\right)^{1 / 2}=\mathcal{O}\left(h^{-1 / 2}\right) .
$$

Proof. By the definitions of $u \equiv u_{h}$ and $f$, it is easy to obtain

$$
\left\|u_{h}^{\prime}\right\|_{L^{2}(J)}^{2}=h^{-2} T, \quad g_{i}= \begin{cases}0, & i=1, \cdots, n-1, \\ 1 / 2, & i=n .\end{cases}
$$

On the other hand, $\left\|f_{h}\right\|_{L^{2}(J)}$ is given as follows

$$
\left\|f_{h}\right\|_{L^{2}(J)}^{2}=\left(f_{h}, f_{h}\right)_{L^{2}(J)}=\left(f, f_{h}\right)_{L^{2}(J)}=g^{T} b=b_{n} / 2,
$$

where $b_{n}$ can be obtained by solving the linear system $L b=g$. Note that $L^{-1}$ is explicitly computed [8]. Indeed, setting the sequences $\left\{p_{i}\right\}$ and $\left\{q_{i}\right\}$ by

$$
p_{0}=1, \quad p_{1}=2, \quad p_{i+1}=4 p_{i}-p_{i-1}, \quad(i=1,2, \cdots),
$$

and

$$
q_{0}=1, \quad q_{1}=4, \quad q_{i+1}=4 q_{i}-q_{i-1}, \quad(i=1,2, \cdots),
$$

respectively, we have

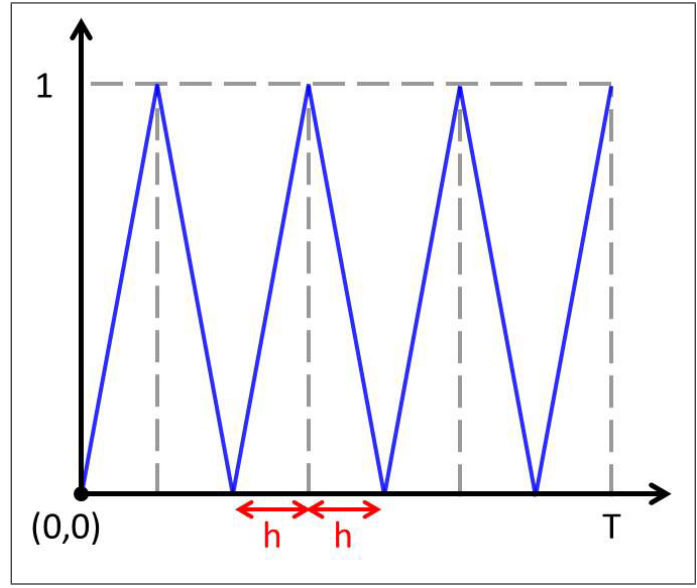

Fig. 2. $u(t)(n=7)$.

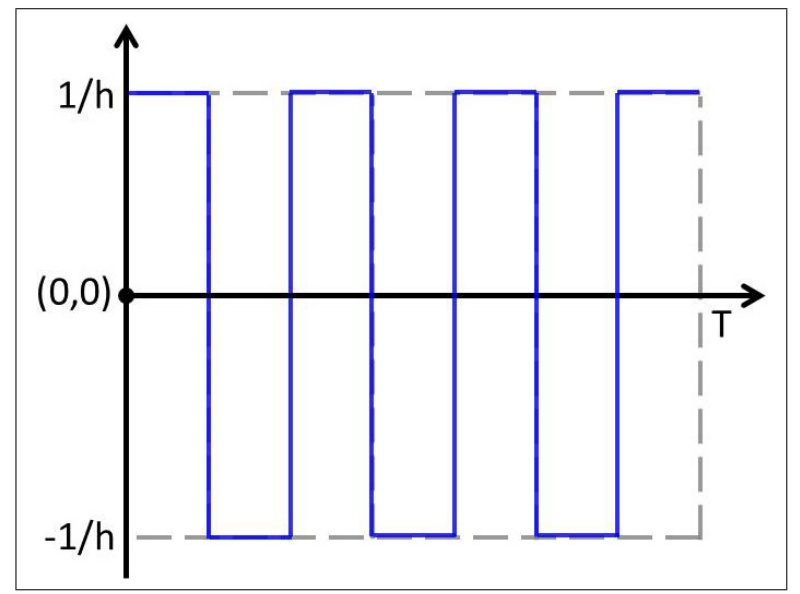

Fig. 3. $f(t)(n=7)$. 


$$
\left(L^{-1}\right)_{i j}=\frac{6}{p_{n} h}(-1)^{i+j} \times \begin{cases}p_{n-j} q_{i-1}, & i \leq j \\ p_{n-i} q_{j-1}, & i \geq j .\end{cases}
$$

Here, (18) can be easily proved by using the fact that $\left\{p_{i}\right\},\left\{q_{i}\right\}$ satisfy

$$
\begin{gathered}
p_{i+1}=2 q_{i}-q_{i-1}, \quad(i \geq 1), \\
p_{j-i} q_{i}-p_{j-i-1} q_{i-1}=p_{j-i+1} q_{i-1}-p_{j-i} q_{i-2}, \quad(i \geq 2, \quad j \geq i+1) .
\end{gathered}
$$

From (17) and (18), we have,

$$
\left\|f_{h}\right\|_{L^{2}(J)}^{2}=\frac{b_{n}}{2}=\frac{1}{2}\left(L^{-1} g\right)_{n}=\frac{1}{4} \frac{6}{h} \frac{q_{n-1}}{p_{n}} .
$$

By $p_{i+1}=2 q_{i}-q_{i-1}$, we obtain

$$
\left\|f_{h}\right\|_{L^{2}(J)}^{2}=\frac{3}{2 h} \frac{q_{n-1}}{2 q_{n-1}-q_{n-2}} .
$$

Moreover, by using the recurrence relation of $\left\{q_{i}\right\}$, we have

$$
q_{n}=\frac{(2+\sqrt{3})^{n+1}-(2-\sqrt{3})^{n+1}}{2 \sqrt{3}} .
$$

Thus we have

$$
\begin{aligned}
\frac{\left\|u_{h}^{\prime}\right\|_{L^{2}(J)}^{2}}{\left\|f_{h}\right\|_{L^{2}(J)}^{2}} & =h^{-2} T\left(\frac{3}{2 h} \frac{q_{n-1}}{2 q_{n-1}-q_{n-2}}\right)^{-1} \\
& =\frac{2 T}{3 h}\left(\frac{q_{n-1}}{2 q_{n-1}-q_{n-2}}\right)^{-1} \\
& =\frac{2 T}{\sqrt{3} h} \frac{(2+\sqrt{3})^{n}+(2-\sqrt{3})^{n}}{(2+\sqrt{3})^{n}-(2-\sqrt{3})^{n}} .
\end{aligned}
$$

Since $h \rightarrow 0$ is equivalent to $n \rightarrow \infty$, we have

$$
\frac{(2+\sqrt{3})^{n}+(2-\sqrt{3})^{n}}{(2+\sqrt{3})^{n}-(2-\sqrt{3})^{n}} \rightarrow 1 \quad \text { as } \quad n \rightarrow \infty
$$

which yields the desired conclusion $\left\|u_{h}^{\prime}\right\|_{L^{2}(J)} /\left\|f_{h}\right\|_{L^{2}(J)}=\mathcal{O}\left(h^{-1 / 2}\right)$.

\subsection{For the case: $\kappa \neq 0$}

In case that $\kappa \neq 0$, by applying Theorem 4.1, we obtain the following result.

Theorem 4.2. For the functions $u$ and $f$ defined by (15), it holds that

$$
\frac{\left\|u_{h}^{\prime}\right\|_{L^{2}(J)}}{\left\|f_{h}\right\|_{L^{2}(J)}}=\mathcal{O}\left(h^{-1 / 2}\right)
$$

Proof. By the fact that $u \in S_{h}$ and taking notice of the definition (8) of $u_{h}$, we also have $u_{h}=u$, which yields

$$
\left\|u_{h}^{\prime}\right\|_{L^{2}(J)}^{2}=h^{-2} T \quad \text { and } \quad\left\|u_{h}\right\|_{L^{2}(J)}^{2}=T / 3 .
$$

And by the definition (15) of the function $f$, we have

$$
\begin{aligned}
\left\|f_{h}\right\|_{L^{2}(J)} & =\left\|P_{h}^{0}\left(u^{\prime}\right)+\kappa u_{h}\right\|_{L^{2}(J)} \\
& \leq\left\|P_{h}^{0}\left(u^{\prime}\right)\right\|_{L^{2}(J)}+\left\|\kappa u_{h}\right\|_{L^{2}(J)} .
\end{aligned}
$$


Thus, we obtain

$$
\begin{aligned}
\frac{\left\|u_{h}^{\prime}\right\|_{L^{2}(J)}}{\left\|f_{h}\right\|_{L^{2}(J)}} & \geq \frac{\left\|u_{h}^{\prime}\right\|_{L^{2}(J)}}{\left\|P_{h}^{0}\left(u^{\prime}\right)\right\|_{L^{2}(J)}+\left\|\kappa u_{h}\right\|_{L^{2}(J)}} \\
& =\frac{\left\|u_{h}^{\prime}\right\|_{L^{2}(J)}}{\left\|P_{h}^{0}\left(u^{\prime}\right)\right\|_{L^{2}(J)}+|\kappa| \sqrt{T / 3}} .
\end{aligned}
$$

Here, from the proof of Theorem 4.1, we have $\frac{\left\|u_{h}^{\prime}\right\|_{L^{2}(J)}}{\left\|P_{h}^{0}\left(u^{\prime}\right)\right\|_{L^{2}(J)}}=\mathcal{O}\left(h^{-1 / 2}\right)$.

Hence we conclude that $\frac{\left\|u_{h}^{\prime}\right\|_{L^{2}(J)}}{\left\|f_{h}\right\|_{L^{2}(J)}}$ is at least $\mathcal{O}\left(h^{-1 / 2}\right)$.

$\mathcal{O}\left(h^{-1 / 2}\right)$ is suited to the numerical results in section 3.1.

\section{Concluding remark}

As described in the remark 3.2 , the matrix norm $\left\|L^{T / 2} G^{-T} D G^{-1} L^{1 / 2}\right\|_{2}^{1 / 2}$ gives the best estimate for $C(h)$ in (10). However, Theorem 4.2 states that $C(h)$ has the order of magnitude with greater than or equals to $\mathcal{O}\left(h^{-1 / 2}\right)$. It proves non-existence of the constant $C$ in (4), i.e., independent of $h$, while $C^{*}$ is given by Lemma 2.2. Particularly, it should be noted that a kind of stability of solutions for the infinite dimensional problem (7) is missing in the finite dimensional scheme (8), even if such a discretization seems to be quite natural.

\section{Acknowledgments}

The authors are very grateful to two anonymous reviewers for their helpful suggestions. We would also like to thank Dr. Xuefeng Liu for his kind help to revise the English expressions in our manuscript. This work was supported by the Grant-in-Aid from the Ministry of Education, Culture, Sports, Science and Technology of Japan (No. 20224001 and No. 23740074) and supported by Kyoto University Mathematics Global COE Program.

\section{References}

[1] T. Kinoshita, T. Kimura, and M.T. Nakao, "A posteriori estimates of inverse operators for initial value problems in linear ordinary differential equations," Journal of Computational and Applied Mathematics, vol. 236, no. 6, pp. 1622-1636, 2011.

[2] M.T. Nakao and K. Hashimoto, "A numerical verification method for solutions of nonlinear parabolic problems," Journal of Math-for-industry, vol. 1, pp. 69-72, 2009.

[3] M.T. Nakao, T. Kinoshita, and T. Kimura, "On a posteriori estimates of inverse operators for linear parabolic initial-boundary value problems," Computing, vol. 94, pp. 151-162, 2012.

[4] S.M. Rump, "Verified bounds for singular values, in particular for the spectral norm of a matrix and its inverse," BIT Numerical Mathematics, vol. 51, no. 2, pp. 367-384, 2011.

[5] S.M. Rump, INTLAB-INTerval LABoratory, in Developments in Reliable Computing, Tibor Csendes, ed., pp. 77-104, Kluwer Academic Publishers, Dordrecht, 1999. http://www.ti3.tu-harburg.de/rump/intlab/

[6] Y. Watanabe, T. Kinoshita, and M.T. Nakao, "A posteriori estimates of inverse operators for boundary value problems in linear elliptic partial differential equations," to appear in Mathematics of Computation.

[7] N. Yamamoto and M.T. Nakao, "Numerical verifications of solutions for elliptic equations in nonconvex polygonal domains," Numerische Mathematik, vol. 65, pp. 503-521, 1993.

[8] T. Yamamoto and Y. Ikebe, "Inversion of band matrices," Linear Algebra Appl., vol. 24, pp. 105111, 1979. 